\title{
Aberrant immunoglobulin class switch recombination and switch translocations in activated B cell-like diffuse large B cell lymphoma
}

Georg Lenz, ${ }^{1}$ Inga Nagel, ${ }^{5}$ Reiner Siebert, ${ }^{5}$ Anna V. Roschke, ${ }^{3}$ Warren Sanger, ${ }^{6}$ George W. Wright, ${ }^{4}$ Sandeep S. Dave, ${ }^{1}$ Bruce Tan, ${ }^{1}$ Hong Zhao, ${ }^{1}$ Andreas Rosenwald, ${ }^{7}$ Hans Konrad Muller-Hermelink, ${ }^{7}$ Randy D. Gascoyne, ${ }^{8}$ Elias Campo, ${ }^{9}$ Elaine S. Jaffe, ${ }^{2}$ Erlend B. Smeland, ${ }^{10}$ Richard I. Fisher, ${ }^{11,12}$ W. Michael Kuehl, ${ }^{3}$ Wing C. Chan, ${ }^{6}$ and Louis M. Staudt ${ }^{1}$
CORRESPONDENCE

Louis M. Staudt:

Istaudt@mail.nih.gov

\footnotetext{
Abbreviations used: $\mathrm{ABC}$, activated B cell-like; AID, activation-induced cytidine deaminase; CSR, class switch recombination; DLBCL, diffuse large B cell lymphoma; FISH, fluorescence in situ hybridization; $\mathrm{GCB}$, germinal center $\mathrm{B}$ cell-like; MALT, mucosaassociated lymphoid tissue; PMBL, primary mediastinal B cell lymphoma.
}

\author{
${ }^{1}$ Metabolism Branch, ${ }^{2}$ Laboratory of Pathology, ${ }^{3}$ Genetics Branch, Center for Cancer Research, and ${ }^{4}$ Biometric Research \\ Branch, Division of Cancer Treatment and Diagnosis, National Cancer Institute (NCI), National Institutes of Health (NIH), \\ Bethesda, MD 20892 \\ 5Institute of Human Genetics, University Hospital Schleswig-Holstein, Campus Kiel, D-24105 Kiel, Germany \\ ${ }^{6}$ Department of Pathology and Department of Microbiology, University of Nebraska Medical Center, Omaha, NE 68198 \\ 7Department of Pathology, University of Würzburg, 97070 Würzburg, Germany \\ ${ }^{8}$ British Columbia Cancer Agency, Vancouver, British Columbia V5Z 4E6, Canada \\ ${ }^{9}$ Hospital Clinic, University of Barcelona, 08007 Barcelona, Spain \\ ${ }^{10}$ Department of Immunology, Rikshospitalet-Radiumhospitalet Medical Center, 0805 Oslo, Norway \\ "11Southwest Oncology Group, San Antonio, TX 78245 \\ 12James P. Wilmot Cancer Center, University of Rochester School of Medicine, Rochester, NY 14627
}

To elucidate the mechanisms underlying chromosomal translocations in diffuse large B cell lymphoma (DLBCL), we investigated the nature and extent of immunoglobulin class switch recombination (CSR) in these tumors. We used Southern blotting to detect legitimate and illegitimate CSR events in tumor samples of the activated $B$ cell-like (ABC), germinal center $B$ cell-like (GCB), and primary mediastinal $B$ cell lymphoma (PMBL) subgroups of DLBCL. The frequency of legitimate CSR was lower in ABC DLBCL than in GCB DLBCL and PMBL. In contrast, $A B C$ DLBCL had a higher frequency of internal deletions within the switch $\mu(\mathrm{S} \mu)$ region compared with GCB DLBCL and PMBL. ABC DLBCLs also had frequent deletions within $S \gamma$ and other illegitimate switch recombinations. Sequence analysis revealed ongoing $S \mu$ deletions within ABC DLBCL tumor clones, which were accompanied by ongoing duplications and activation-induced cytidine deaminase-dependent somatic mutations. Unexpectedly, short fragments derived from multiple chromosomes were interspersed within $S \mu$ in one case. These findings suggest that ABC DLBCLs have abnormalities in the regulation of CSR that could predispose to chromosomal translocations. Accordingly, aberrant switch recombination was responsible for translocations in ABC DLBCLs involving $B C L 6, M Y C$, and a novel translocation partner, SPIB.

Diffuse large B cell lymphoma (DLBCL), the most common type of non-Hodgkin's lymphoma, accounts for $\sim 30 \%$ of all lymphoma cases (1). DLBCL can be cured using anthracycline-based chemotherapy regimens in just $40-50 \%$ of patients, suggesting that DLBCL represents a heterogeneous disease (2). This concept was supported by several recent gene

$\overline{\text { The online version of this article contains supplemental material. }}$ expression profiling studies distinguishing at least three different molecular DLBCL subgroups (3-7). These three subgroups have been termed germinal center B cell-like (GCB) DLBCL, activated B cell-like (ABC) DLBCL, and primary mediastinal B cell lymphoma (PMBL). The DLBCL subgroups differ not only with respect to their gene expression profiles, but they also have significantly different overall survival rates and use distinct oncogenic pathways (8). 
By these criteria, the DLBCLs can be viewed as distinct diseases that arise from B lymphocytes at different stages of differentiation.

GCB DLBCL tumors express many genes that are characteristic for normal germinal center B cells, suggesting that this lymphoma subgroup arises from B cells at the germinal center stage of differentiation, probably from the highly proliferative centroblasts $(3,4,6)$. Characteristically, GCB DLBCLs express the key transcriptional repressor BCL6 and consequently lack expression of its target genes $(9,10)$.

In contrast, the gene expression profile of ABC DLBCL suggests that this lymphoma subgroup is derived from B cells that are in the process of differentiating from germinal center $\mathrm{B}$ cells to plasma cells $(4,6)$. This hypothesis was based on the observation that ABC DLBCLs have down-regulated most of the germinal center $\mathrm{B}$ cell signature genes, including BCL6. Instead, ABC DLBCLs express a key regulator of the secretory phenotype of plasma cells, $X B P-1$, as well as many of its downstream target genes $(6,11)$. Strong support for this hypothesis was provided by the recent demonstration that genomic mutations, deletions, and rearrangements of the gene encoding Blimp-1 are recurrent in ABC DLBCLs but are not present in GCB DLBCLs $(12,13)$. Blimp-1 is the master regulator of plasmacytic differentiation that represses directly or indirectly the entire gene expression program of mature B cells (14). Thus, the inactivation of Blimp-1 in roughly one quarter of ABC DLBCL cases provides direct evidence that a block in differentiation is a key event in the pathogenesis of this lymphoma type.

The third subgroup, PMBL, has a characteristic gene expression signature that distinguishes it from GCB DLBCL and $A B C$ DLBCL, but which it shares with the malignant cells of Hodgkin lymphoma (5, 7). PMBL characteristically arises in the mediastinum and, upon pathological examination, a thymic remnant can often be found associated with the tumor mass. This finding suggests that PMBL arises from a rare $B$ cell subpopulation that resides in the thymus (15). PMBLs express some genes in common with GCB DLBCLs, including BCL6, but do not express the ABC DLBCL signature genes and, particularly, do not express genes associated with plasmacytic differentiation (8).

An important event after activation of mature B cells by antigen is Ig class switch recombination (CSR), which can occur within the germinal center or during the extrafollicular activation of $\mathrm{B}$ cells. CSR is a recombination and deletion mechanism that juxtaposes a downstream Ig heavy chain $\left(\mathrm{C}_{\mathrm{H}}\right)$ segment to the rearranged $\mathrm{V}_{\mathrm{H}} \mathrm{D}_{\mathrm{H}} \mathrm{J}_{\mathrm{H}}$ segment, thereby switching the Ig isotype of a B cell from IgM to IgG, IgA, or IgE. CSR occurs within the highly repetitive switch regions located $5^{\prime}$ of each constant region $(16,17)$. The enzyme activation-induced cytidine deaminase (AID) is the B cellspecific factor that is absolutely required for CSR (18-22). In the current understanding of CSR, AID initiates the reaction by creating double-strand DNA breaks in the switch regions. These double-strand DNA breaks recruit and activate DNA damage response proteins, including ataxia-teleangiectasia, histone $\mathrm{H} 2 \mathrm{AX}$, and 53BP1, which coordinate a ligation reaction that uses the enzymatic machinery of the nonhomologous end joining reaction (23-27). CSR is precisely regulated by extracellular signals that influence the choice of which downstream switch regions are involved in the reaction. This regulation is achieved by the production of sterile mRNA transcripts that traverse a switch region and facilitate the recruitment and action of AID (17).

The elaborate controls governing CSR can, however, go awry as indicated by the frequent subversion of CSR to create aberrant switch translocations in multiple myeloma and other lymphoid malignancies (28). Indeed, experimental overexpression of AID can directly promote chromosomal translocations between the Ig heavy chain locus and $c-M Y C$ in mouse B lymphocytes $(29,30)$. Interestingly, AID is one of the few germinal center genes that is more highly expressed in ABC DLBCL than in GCB DLBCL (6), which may be due to the high expression of IRF4 $(3,4,6)$, a transcription factor that directly or indirectly increases AID expression $(31,32)$.

Given the high expression of AID in ABC DLBCL and the ability of AID to promote aberrant class switch translocations, we investigated the nature and extent of CSR events in this lymphoma type in comparison to other subgroups of DLBCL. To this end, we probed the genomic structure of the Ig switch regions by Southern blotting in 22 DLBCL cell lines and 92 DLBCL patient samples, which revealed significant differences in the frequency of CSR between the three subgroups. Unexpectedly, we discovered an abnormality in switch recombination in ABC DLBCLs that led to frequent nonproductive intra-switch CSR, insertion of foreign chromosomal DNA into the Ig locus, and chromosomal translocations involving the switch regions.

\section{RESULTS}

Class switch recombination events in subgroups of DLBCL

To investigate the frequency and nature of CSR events in tumors of different DLBCL subgroups, we used a Southern blot assay that can detect both legitimate CSR events and illegitimate recombination and deletion events involving the Ig switch regions $(28,33)$. This assay uses three different pairs of Southern blot probes that hybridize either $5^{\prime}$ or $3^{\prime}$ of the $\mathrm{S} \mu, \mathrm{S} \gamma$, and $\mathrm{S} \alpha$ switch regions (Fig. 1). With the $\mathrm{S} \mu$ probe pairs, a single hybridizing fragment is present in germ line DNA, whereas the $S \gamma$ probes hybridize to four germ line fragments representing $\mathrm{S} \gamma 1, \mathrm{~S} \gamma 2, \mathrm{~S} \gamma 3$, and $\mathrm{S} \gamma 4$, and the $\mathrm{S} \alpha$ probes hybridize to two germ line fragments representing S $\alpha 1$ and S $\alpha 2$. Genomic DNAs from 22 DLBCL cell lines and 92 DLBCL patient samples were digested with either of two restriction enzymes (HindIII or SphI) and subsequently hybridized with the different probes. A switch region in germ line configuration will be reflected by a restriction fragment that hybridizes with the corresponding $5^{\prime}$ and $3^{\prime}$ switch probes and that is also present in the placental control DNA (Fig. 1, A and F). A legitimate switch event, such as between $S \mu$ and $S \gamma$, creates a restriction fragment that will be detected by the $5^{\prime} \mathrm{S} \mu$ and the $3^{\prime} \mathrm{S} \gamma$ probes (Fig. 1, B and F). 


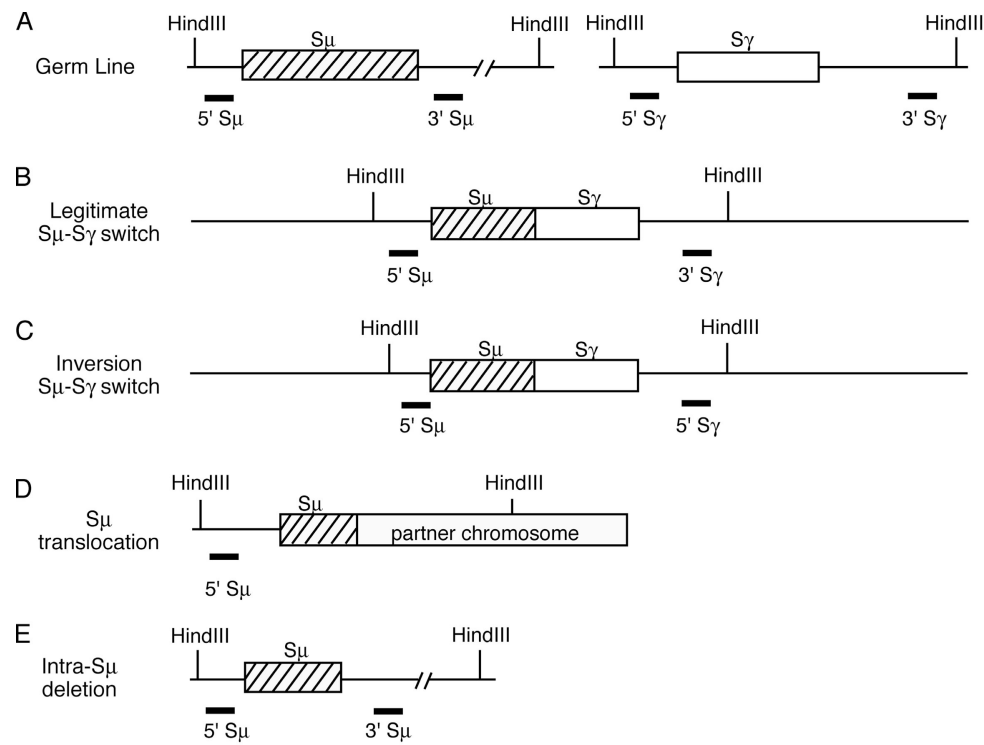

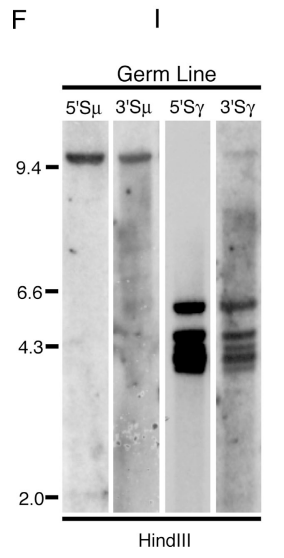

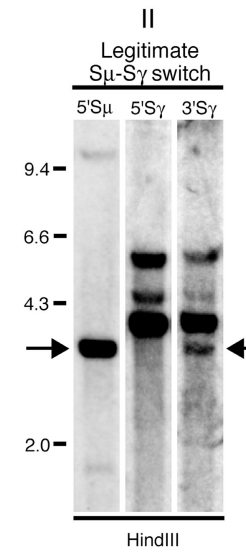

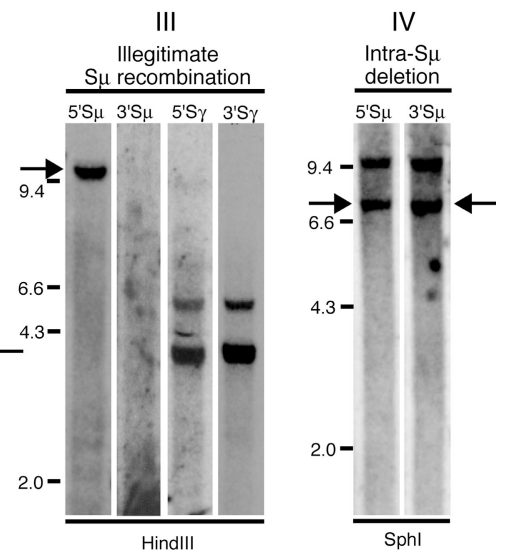

Figure 1. Analysis of class switch recombination by Southern blot. $(A-E)$ The position of Hindlll restriction sites are shown. Thick lines indicate binding sites of respective probes. (A) Germ line configuration of switch $\mu$ and switch $\gamma$ regions. (B) Legitimate switch $\mu$ to $\gamma$, detected by $5^{\prime} S \mu$ and 3' S $\gamma$ probe. (C) Inversion switch $\mu$ to $\gamma$, detected by $5^{\prime} S \mu$ and $5^{\prime}$ S $\gamma$ probe. (D) Chromosomal translocation, detected by $5^{\prime} \mathrm{S} \mu$ probe. (E) Intra-switch $\mu$ deletion, detected by $5^{\prime} S \mu$ and 3' $S \mu$ probe. (F) Applied restriction enzyme

Legitimate switch recombinations may also occur between downstream switch regions, such as between $\mathrm{S} \gamma$ and $\mathrm{S} \alpha$. Inversion CSR events, such as between $S \mu$ and $S \gamma$, generate a restriction fragment that either hybridizes with the $5^{\prime}$ switch $\mu$ and $\gamma$ probes or with the two $3^{\prime}$ switch probes (Fig. 1 C). An illegitimate switch recombination event is operationally defined by a restriction fragment that hybridizes with only one switch region probe (Fig. 1 F). This may be caused by a chromosomal translocation (Fig. $1 \mathrm{D}$ ), an insertion of foreign sequences into a switch region, a polymorphism or mutation of the restriction enzyme binding site, or an atypical deletion within the Ig heavy chain locus. Intra-switch recombination events may occur in which part of a switch region is deleted without resulting in legitimate switch recombination, and these can be detected reliably when they occur in $\mathrm{S} \mu$ (Fig. 1,

is indicated below lanes. The probes used for hybridization are shown above each lane. Panel I: Restriction digest of placental DNA shows germ line configuration. Panel II: Restriction digest of patient sample 529 genomic DNA. Arrows indicate legitimate switch recombination $\mu$ to $\gamma$. Panel III: Restriction digest of SUDHL-2 genomic DNA. Arrow indicates illegitimate switch recombination detected by 5' $S \mu$ probe. Panel IV: Restriction digest of patient sample 1002 genomic DNA. Arrows indicate intra-switch $\mu$ deletion.

$\mathrm{E}$ and $\mathrm{F})$. The human Ig locus contains four $\mathrm{S} \gamma$ and two $\mathrm{S} \alpha$ regions. CSR can potentially join different $\mathrm{S} \gamma$ regions or different $\mathrm{S} \alpha$ regions together. Using the Southern blot assay, these events would be impossible to distinguish from intra-S $\gamma$ deletions and intra-S $\alpha$ deletions, respectively.

The analysis of 6 ABC DLBCL and 16 GCB DLBCL cell lines demonstrated differences in the frequency of legitimate switch recombination events (Table S1, available at http://www.jem.org/cgi/content/full/jem.20062041/DC1). Whereas none of the ABC DLBCL cell lines had a germ line configuration of a switch region, four (25\%) of the GCB DLBCL lines had only germ line switch regions. 1 ABC DLBCL cell line (17\%) had a legitimate switch involving $\mathrm{S} \mu$ and S $\gamma$, whereas 11 GCB DLBCL cell lines (69\%) had a legitimate switch from $S \mu$ to either $S \gamma$ or $S \alpha$ or from $S \alpha$ to $S \gamma$. 
Illegitimate CSR events were detected in half of the ABC DLBCL and GCB DLBCL cell lines.

Analysis of 92 primary biopsy samples revealed differences between ABC DLBCL and GCB DLBCL with respect to the nature and extent of CSR (Fig. 2 and Table S2, which is available at http://www.jem.org/cgi/content/full/ jem.20062041/DC1). Among 50 ABC DLBCL cases, the switch regions were in germ line configuration in only 4 (8\%). Only 15 cases (30\%) had a legitimate switch recombination. 28 cases (56\%) showed an illegitimate switch recombination event, and 6 cases $(12 \%)$ had an inversion switch recombination between $\mathrm{S} \mu$ and $\mathrm{S} \gamma$ or $\mathrm{S} \alpha$ (5 cases with inversion switch recombination between $\mathrm{S} \mu$ and $\mathrm{S} \gamma$, and 1 case with inversion switch recombination between $\mathrm{S} \mu$ and $\mathrm{S} \alpha$ ). Strikingly, 24 ABC DLBCLs (48\%) had intra-S $\mu$ deletion events (Fig. 1, E and F), and 64\% of cases showed a deletion/recombination in $\mathrm{S} \gamma$.

As with ABC DLBCLs, only a small fraction of GCB DLBCLs had switch regions in the germ line configuration (4 out of 31 cases; 13\%). However, legitimate CSR events were more common in GCB DLBCLs, occurring in 18 of 31 cases tested (58\%) compared with ABC DLBCLs $(\mathrm{P}=0.012)$. Conversely, GCB DLBCLs had lower frequencies of several aberrant class switch events compared with ABC DLBCLs, including illegitimate switch recombination $(32 \% ; \mathrm{P}=$ $0.037)$, inversion switch recombination $(0 \% ; \mathrm{P}=0.045)$, intra-S $\mu$ deletion $(13 \% ; \mathrm{P}=0.0012)$, and intra-S $\gamma$ deletion/ recombination $(29 \% ; \mathrm{P}=0.002)$.

Of 11 PMBL cases available for analysis, 7 (64\%) had legitimate CSR events, which was significantly more than among ABC DLBCLs $(\mathrm{P}=0.035)$. Illegitimate CSR events were detected in five cases (45\%), and inversion switch events were detected in three cases (27\%). In contrast to ABC DLBCLs,
intra-S $\mu$ deletions were not present in any PMBL patient sample analyzed $(\mathrm{P}=0.003)$.

As a control, we investigated CSR events in mucosaassociated lymphoid tissue (MALT) lymphoma because this postgerminal center lymphoma type has been reported to have aberrant CSR events in some cases (34). Like ABC DLBCL, MALT lymphomas had a relatively low frequency of legitimate CSR events (6/17 cases investigated; 35\%). However, in contrast to ABC DLBCLs, none of the MALT lymphomas had intra-S $\mu$ deletions $(\mathrm{P}=0.0004)$. MALT lymphomas also had fewer illegitimate CSR events than ABC DLBCLs (29\%; $\mathrm{P}=0.058)$ and intra-S $\gamma$ deletions/ recombinations $(29 \% ; \mathrm{P}=0.013)$.

In summary, ABC DLBCLs had a lower frequency of legitimate class switch recombination events relative to the other lymphoma subtypes and a higher frequency of intra-S $\mu$ deletion, intra-Sy deletion/recombination events as well as illegitimate switch recombination events. Intra-S $\alpha$ deletion/ recombination events were detected, but there were no significant differences in frequency of these events among the lymphoma subtypes (Table S2).

\section{Expression of $A I D$ in lymphoma subsets}

AID mRNA levels were assessed in 245 primary lymphoma specimens with the use of Affymetrix oligonucleotide microarrays (Fig. 3 A). Highest levels of AID expression were observed in ABC DLBCL and PMBL, and these lymphoma types had, respectively, threefold and 2.5-fold higher expression than GCB DLBCL (ABC DLBCL vs. GCB DLBCL, $\mathrm{P}=0.00006$; PMBL vs. GCB DLBCL, $\mathrm{P}=0.01)$. The pregerminal center lymphoma types, mantle cell lymphoma and small lymphocytic lymphoma, both had low levels of $A I D$ expression compared with any of the DLBCL subgroups as

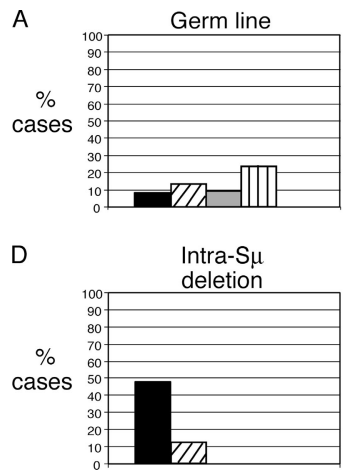

Figure 2. Summary of the analysis of class switch recombination in 92 DLBCL and 17 MALT lymphoma patients. (A) Germ line. No significant differences can be observed between the different lymphoma subtypes. (B) Legitimate switch recombination. GCB DLBCL and $P M B L$ show a significantly higher frequency of legitimate switch recombinations compared with $A B C D L B C L$ ( $A B C$ DLBCL vs. GCB $D L B C L, P=0.012 ; A B C D L B C L$ vs. $P M B L, P=0.035)$. (C) Inversion switch recombination. $A B C D L B C L$ shows a significantly higher rate of inversion switch recombinations compared with GCB DLBCL (ABC DLBCL vs. GCB DLBCL, $P=0.045$ ). (D) Intra-switch $\mu$ deletions. ABC DLBCL shows a significantly higher rate of intra-switch $\mu$ deletions compared with GCB DLBCL, PMBL, or MALT (ABC DLBCL vs. GCB DLBCL, P = 0.0012; $A B C D L B C L$ vs. $P M B L, P=0.003 ; A B C D L B C L$ vs. MALT, $P=0.0004)$. (E) Intra-switch $\gamma$ deletion/recombination. ABC DLBCL shows a significantly higher rate of intra-switch $\gamma$ deletion/recombination compared with GCB DLBCL and MALT (ABC DLBCL vs. GCB DLBCL, P = 0.002; $A B C D L B C L$ vs. MALT, $P=0.013)$. (F) Illegitimate switch recombination. $A B C D L B C L$ shows a significantly higher rate of illegitimate switch recombinations compared with GCB DLBCL (ABC DLBCL vs. GCB DLBCL, $P=0.037)$. 
did the post-germinal center entity, MALT lymphoma (ABC DLBCL vs. mantle cell lymphoma, $\mathrm{P}=2.2 \times 10^{-16}$; ABC DLBCL vs. small lymphocytic lymphoma, $\mathrm{P}=0.0003$; ABC DLBCL vs. MALT, $\mathrm{P}=2.16 \times 10^{-5}$ ).

Having identified a small subset of DLBCLs in which the Ig switch regions were in germ line configuration, we investigated whether these lymphomas might have lower levels of AID expression. Indeed, among ABC DLBCLs, cases with germ line switch regions had eightfold lower expression compared with non-germ line cases (Fig. $3 \mathrm{~B}$; P = 0.0012). Likewise, those GCB DLBCLs with germ line switch regions had lower AID expression than their non-germ line counterparts $(\mathrm{P}=0.09)$.

\section{Structure of intra-S $\mu$ deletions}

To characterize intra-S $\mu$ deletions further, we amplified the $\mathrm{S} \mu$ regions from four ABC DLBCL cases (cases 756, 692, 645 , and 428) that had putative intra-S $\mu$ deletions based on the Southern blot assay. All intra-S $\mu$ deletions were confirmed by PCR (Fig. 4 A). Unexpectedly, however, case 428, which had a single intra-S $\mu$ deletion band using the Southern blot assay, yielded a cluster of bands ranging from $\sim 2.7$ to $\sim 3.9 \mathrm{~kb}$ in size after PCR for the $\mathrm{S} \mu$ region. This finding suggested that there might be intra-clonal variation in the structure of the intra-S $\mu$ deletions.

To examine this possibility, we cloned the amplified intra-S $\mu$ deletion bands of the four ABC DLBCL samples and determined the sequence of three to five clones per case. The molecular clones derived from a single case were remarkably diverse, showing 1-19 separate deletion events within $\mathrm{S} \mu$ (Table I, Fig. $4 \mathrm{~B}$, and Fig. S1, which is available at http://www.jem.org/cgi/content/full/jem.20062041/DC1). Even in cases in which the $\mathrm{S} \mu \mathrm{PCR}$ product appeared to be a single band, the clones obtained were diverse in sequence.

We next examined the $5^{\prime}$ end of the amplified $\mathrm{S} \mu$ region in detail for the presence of somatic mutations. From each case, we obtained molecular clones that had a large number of somatic mutations (Table I, Fig. 4 B, and Fig. S1). These mutations occurred most frequently at RGYW motifs, strongly suggesting that they were caused by AID (Fig. S2,
Table I. Sequence analysis of $\mathrm{S} \mu$ deletions in $A B C D L B C L$ and GCB DLBCL patient samples

\begin{tabular}{lcccc}
\hline Sample & $\begin{array}{c}\text { DLBCL } \\
\text { subtype }\end{array}$ & $\begin{array}{c}\text { No. of } \mathrm{S} \mu \\
\text { deletions }\end{array}$ & $\begin{array}{c}\text { No. of somatic } \\
\text { mutations }^{\mathrm{a}}\end{array}$ & $\begin{array}{c}\text { No. of } \\
\text { duplications }\end{array}$ \\
\hline 428 & ABC DLBCL & 19 & n.a. & 0 \\
645 & ABC DLBCL & 13 & n.a. & 3 \\
692 & ABC DLBCL & 9 & n.a. & 2 \\
756 & ABC DLBCL & 5 & 3 & 0 \\
459 & GCB DLBCL & 11 & 3 & 0 \\
742 & GCB DLBCL & 6 & 6 & 1
\end{tabular}

n.a., not available, as investigated 250-bp segment partly or completely deleted. aSomatic mutations determined in 250-bp segment at 5' end of $\mathrm{S} \mu$.

available at http://www.jem.org/cgi/content/full/jem $.20062041 / \mathrm{DC} 1)$. In some instances, different molecular clones from the same case had distinct somatic mutations whereas other molecular clones had no mutations.

As a control, we performed a similar sequence analysis of the $S \mu$ region in two ABC DLBCL cases in which $S \mu$ was in germ line configuration by Southern blot analysis. As expected, no deletion was detected in the amplified $\mathrm{S} \mu$ regions from these cases by PCR. Further, these two cases had significantly fewer somatic mutations in $\mathrm{S} \mu$ than ABC DLBCL cases with intra-S $\mu$ deletions.

In addition to deletions and mutations, sequence analysis revealed several duplications of $\mathrm{S} \mu$ segments of up to $40 \mathrm{bp}$ in length (Table I and Fig. S1). Unexpectedly, we also detected insertions of short DNA segments derived from other chromosomes into the $\mathrm{S} \mu$ region of one of the four $\mathrm{ABC}$ DLBCL cases analyzed (Fig. 4, B and C). These insertions, which varied in length from 146 to $160 \mathrm{bp}$, were derived from chromosomes 17,12 , and 4 and were arranged in tandem with no gaps between the insertions (Fig. 4 C). These segments were situated within a $\mathrm{S} \mu$ deletion of $10 \mathrm{bp}$. Interestingly, one of the three clones analyzed from this case had a 19-bp deletion within the inserted chromosome 4 sequence (Fig. 4 B). This finding suggests that AID is able to create breaks and deletions in ectopic sequences that are inserted into the $\mathrm{S} \mu$ region.

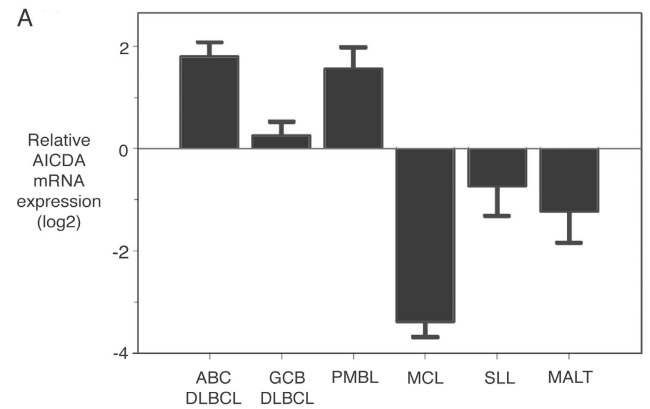

Figure 3. Relative AID mRNA expression in different lymphoma subtypes. (A) $A B C D L B C L$ s and PMBLs express the highest level of $A I D$ mRNA. (B) Comparison of AID mRNA expression in ABC DLBCL and GCB

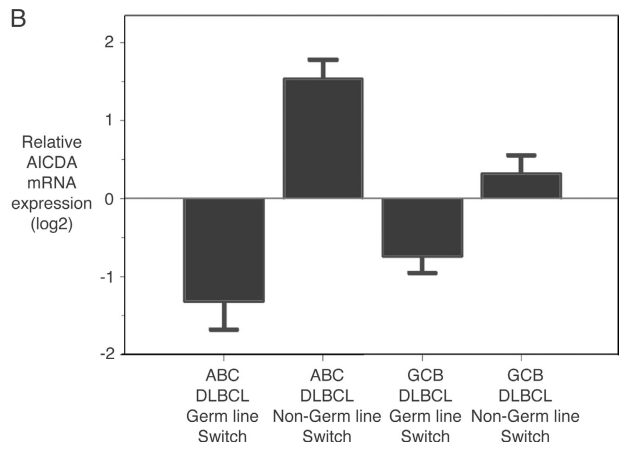

DLBCL germ line cases versus non-germ line cases. Non-germ line cases express higher AID mRNA compared with germ line cases. 

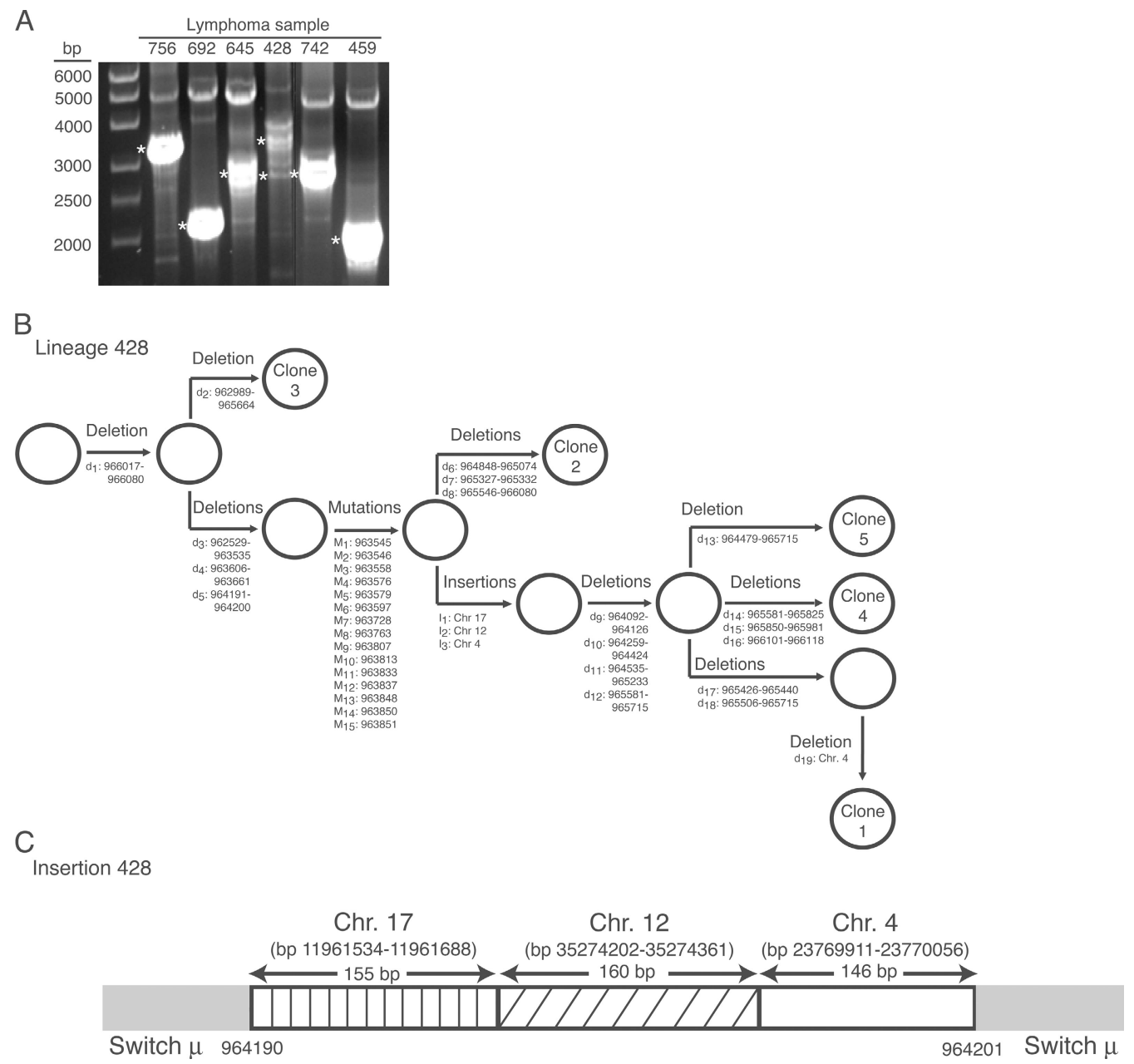

Figure 4. Analysis of intra-switch $\mu$ deletions. (A) PCR amplification of intra-switch $\mu$ deletions (ethidium bromide-stained $0.8 \%$ agarose gel). Asterisks indicate cloned PCR bands for sequence analysis. (B) Lineage model of aberrations in ABC DLBCL patient sample 428. $d$, deletion; $M$, mutation; I, insertion. Open circles represent putative clonal precursors.

For each of the four cases, we were able to organize all of the $S \mu$ deletions, mutations, duplications, and insertions into lineage models of the stepwise accumulation of these events in individual molecular clones (Fig. $4 \mathrm{~B}$ and Fig. S1). The fact that such lineage diagrams can be drawn is consistent with ongoing molecular alterations of the $S \mu$ regions within the malignant clones. In three of four cases, the first abnormality that occurred was one or several internal deletions. Subsequently, each clone underwent several steps of mutations, internal tandem duplications, and insertions of DNA from other chromosomes. Finally, most of the clones sustained one or more $S \mu$ deletions. Thus, multiple molecular mechanisms that perturb $\mathrm{S} \mu$ structure were ongoing and interspersed in time after malignant transformation of a B cell into ABC DLBCL.

Although intra-S $\mu$ deletions were much less frequent in GCB DLBCLs than in ABC DLBCLs (Fig. 2 D), we investigated whether ongoing intra-clonal $\mathrm{S} \mu$ alterations could also occur in GCB DLBCL. To this end, we amplified the $\mathrm{S} \mu$
Numbers refer to GenBank accession number NG_001019.4. (C) Sequence analysis of DNA insertions into $S \mu$ in ABC DLBCL case 428. bp numbers refer to accession number Hs17_10875 for chromosome 17, accession number Hs12_29578 for chromosome 12, and accession number Hs4_16510 for chromosome 4.

regions of two GCB DLBCL patient samples (cases 742 and 459) that had intra-S $\mu$ deletions by the Southern blot assay. In both cases, intra-S $\mu$ deletions were confirmed by PCR (Fig. 4 A). Sequence analysis revealed clonal heterogeneity in both cases, with 6-11 deletions per case, various somatic mutations, and a duplication of $194 \mathrm{bp}$ in GCB DLBCL case 742 (Table I). Thus, GCB DLBCL and ABC DLBCL can apparently use similar aberrant CSR mechanisms, albeit with different frequencies.

\section{Switch translocations in DLBCL}

As assessed by the Southern blotting, ABC DLBCL had the highest frequency of illegitimate switch recombination events (Fig. 2). To determine whether these illegitimate events might represent chromosomal translocations involving the switch regions, we first performed fluorescence in situ hybridization (FISH) using $\mathrm{C}_{\mathrm{H}}$ and $\mathrm{V}_{\mathrm{H}}$ probes to determine the presence of IgH translocations. We investigated 10 DLBCL 
cell lines (2 ABC DLBCL cell lines: OCI-Ly10, and SUDHL2, and 8 GCB DLBCL cell lines: NUDHL-1, NUDUL-1, OCI-Ly1, OCI-Ly2, OCI-Ly-8, SUDHL-4, Toledo, and RCK8) that had an illegitimate switch recombination phenotype by Southern blotting. As a control, we analyzed the ABC DLBCL cell line U2932 that did not show an illegitimate switch recombination. IgH translocations were detected by FISH in all 10 DLBCL cell lines with the illegitimate CSR phenotype, but not in U2932, demonstrating that the majority of illegitimate CSR events represent chromosomal translocations.

We next investigated whether two oncogenes of importance in DLBCL, MYC and BCL6, were involved in illegitimate switch translocations. FISH analyses of DLBCL samples were performed using "break-apart" probes spanning the MYC and BCL6 loci, revealing MYC disruptions in 7\% of cases analyzed $(n=87$; reference 35$)$ and BCL6 disruptions in $19 \%$ of cases analyzed $(n=133$; unpublished data). Some of these positive cases were investigated further using long- distance PCR assays to detect MYC or BCL6 translocations into the $\mathrm{S} \mu$ or $\mathrm{S} \gamma$ regions. These assays revealed an $M Y C$ translocation into the $\mathrm{S} \gamma$ region in one ABC DLBCL case and a BCL6 translocation into the $\mathrm{S} \mu$ region in another (Fig. $5 \mathrm{~A})$. In cell lines with illegitimate switch CSR events, longdistance PCR demonstrated a MYC-S $\gamma$ translocation in the GCB DLBCL cell line NUDUL-1 (Fig. 5 A).

Additionally, we investigated whether a $t(14 ; 19)$ translocation in the ABC DLBCL cell line OCI-Ly3 might be the result of aberrant switch recombination. FISH using a whole chromosome 19 painting probe revealed that OCI-Ly3 cells show a $\mathrm{t}(14 ; 19)$ as well as a complicated translocation involving chromosomes 19, 22, 4, and 18 (unpublished data; Fig. 5 B). A variety of BAC probes from chromosome 19 were used in FISH analyses to narrow the region of chromosome 19 involved in the translocations (unpublished data). Ultimately, three BAC probes in the vicinity of the SPIB gene were identified that clustered around the translocation breakpoint (Fig. 5 B). The $\mathrm{t}(14 ; 19)$ derivative chromosome hybridized
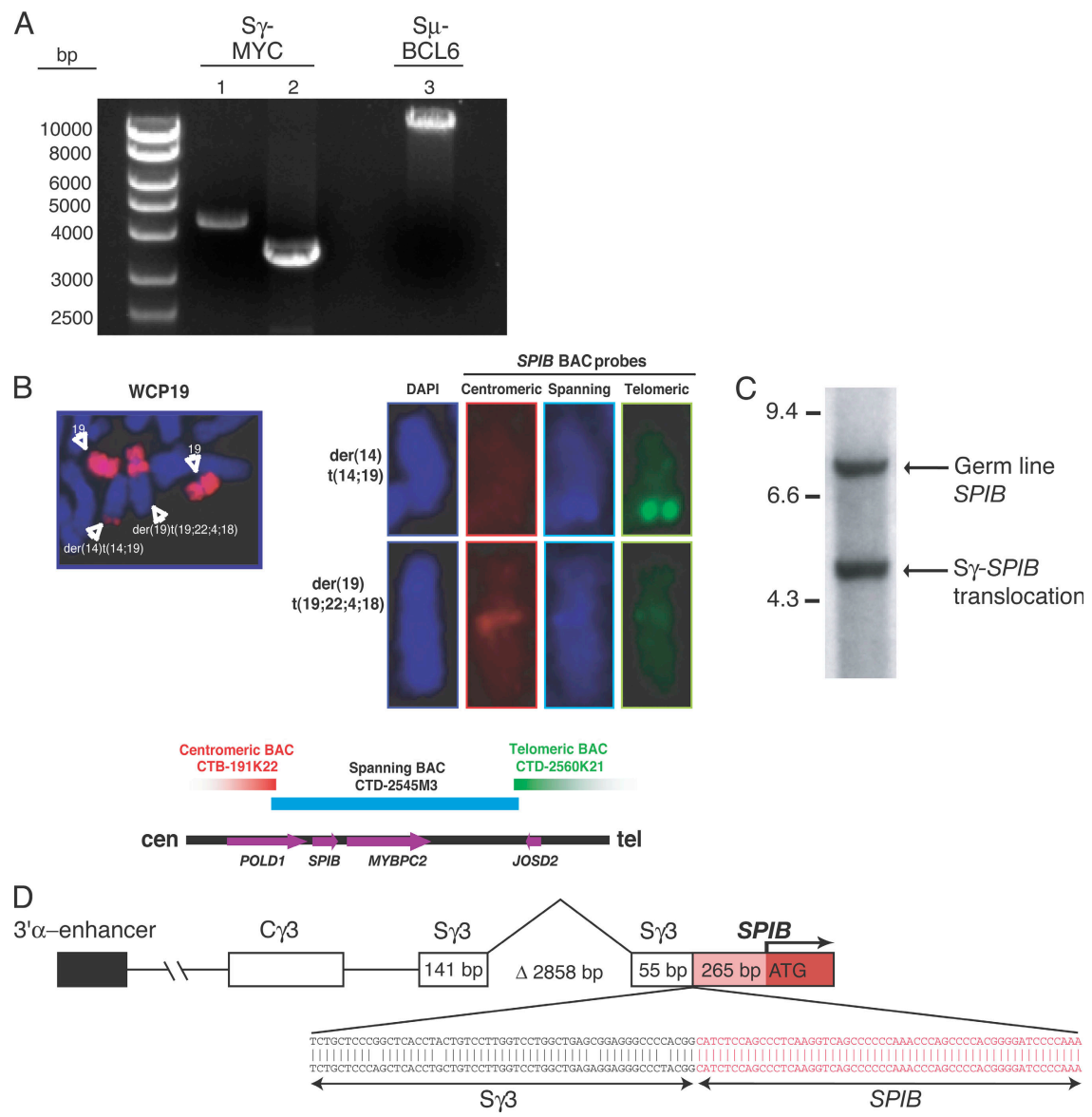

Figure 5. Switch translocations in DLBCL. (A) PCR for detection of $B C L 6 / M Y C$ switch translocations (ethidium bromide-stained $0.8 \%$ agarose gel). Lane 1, S $\gamma$-MYC translocation in ABC DLBCL case 1000; lane 2, S $\gamma-M Y C$ translocation in GCB DLBCL cell line NUDUL- 1 ; lane 3, S $\mu-B C L 6$ translocation in $A B C D L B C L$ case 709. (B) Whole chromosome painting of chromosome 19 detects $t(14 ; 19)$ and $t(19 ; 22 ; 4 ; 18)$. Hybridization of $t(14 ; 19)$ with spanning and telomeric BAC SPIB probes. No hybridization with centromeric BAC probe. Hybridization of $t(19 ; 22 ; 4 ; 18)$ with centromeric and spanning BAC SPIB probe, no hybridization with telomeric probe. (C) Detection of switch $\gamma$-SPIB translocation by Southern blot in OCI-Ly3. (D) Sequence analysis of switch $\gamma$-SPIB translocation. $\Delta$, deletion in $\mathrm{S} \gamma$. 
with BAC probes spanning or telomeric to the SPIB gene, but not with a BAC probe centromeric to SPIB. This suggested that the translocation breakpoint was $5^{\prime}$ of the transcriptional start site of SPIB. In contrast, the $\mathrm{t}(19 ; 22 ; 4 ; 18)$ hybridized with the centromeric BAC probe and weakly with the spanning BAC probe (Fig. 5 B).

We next used Southern blot hybridization to confirm the SPIB translocation that was detected by FISH. With OCI-Ly3 cells, a 332-bp probe binding $\sim 200$ bp $3^{\prime}$ of the start codon of SPIB hybridized to one germ line fragment and to a second smaller fragment (Fig. 5 C), whereas only the germ line fragment was observed in other lymphoma cell lines (unpublished data). Finally, we were able to amplify the translocation breakpoint using PCR primers derived from the $S y$ region and from the $5^{\prime}$ end of SPIB. Sequence analysis confirmed that the breakpoint occurred $265 \mathrm{bp} 5^{\prime}$ of the $S P I B$ transcriptional start site and joined SPIB with the $S \gamma 3$ region (Fig. 5 D). Additionally, the translocated $\mathrm{S} \gamma 3$ region sustained a second deletion of 2,858 bp, demonstrating that the processes responsible for intra-switch deletion and switch translocation can target the same Ig allele (Fig. 5 D). As a consequence of this translocation, the intact SPIB coding region was placed in proximity to the $\operatorname{Ig} 3^{\prime} \alpha$ enhancer (Fig. $5 \mathrm{D})$, resulting in a high level of SPIB mRNA expression compared with most other DLBCL biopsy samples and cell lines (not depicted).

\section{DISCUSSION}

Using a Southern blot assay, we have discovered significant differences in the frequency and character of CSR among three molecular subtypes of DLBCL. Unexpectedly, almost half of the ABC DLBCLs had internal deletions in $S \mu$, events that were present rarely among GCB DLBCLs (13\%) and absent among PMBLs and MALT lymphomas. Furthermore, $64 \%$ of ABC DLBCL cases had abnormal CSR events involving S $\gamma$ regions, which occurred much less commonly in GCB DLBCL (29\%), PMBL (36\%), and MALT lymphoma $(29 \%)$. In normal B cells, intra-S $\mu$ deletion occurs in only $12.5-18 \%$ of cells $(36,37)$, and intra-S $\gamma$ deletion/recombination events are extremely rare (37). Therefore, the high frequency of both intra-S $\mu$ and intra-S $\gamma$ deletion/recombination events in ABC DLBCL strongly suggests that CSR is functionally impaired in this lymphoma type, potentially predisposing this lymphoma type to chromosomal translocations. Conversely, legitimate CSR was significantly less common in ABC DLBCL than GCB DLBCL or PMBL. Consistent with this finding, ABC DLBCLs express IgM mRNA at significantly higher levels than do GCB DLBCLs (6).

Constitutive overexpression of AID seems a likely contributor to the aberrant CSR in ABC DLBCL. Normal B lymphocytes transcriptionally activate $A I D$ upon stimulation by antigen or mitogens, and this up-regulation requires the activity of the transcription factor IRF4 $(31,32)$. ABC DLBCLs have high expression of IRF4 due to constitutive NF- $\kappa B$ pathway activation $(3,4,38,39)$, which may account for the high AID expression in ABC DLBCL despite the fact that most other germinal center B cell signature genes are down-regulated (6). Indeed, of all lymphoma types studied, AID mRNA is highest in ABC DLBCL (Fig. 3) and, consequently, AID protein is more highly expressed in $\mathrm{ABC}$ DLBCL than in GCB DLBCL (40). Interestingly, the few ABC DLBCLs that lacked AID expression were those that had a germ line configuration of the Ig switch regions. This result is consistent with the hypothesis that AID action is required for the aberrant CSR that characterizes the majority of ABC DLBCLs.

In normal $\mathrm{B}$ cell responses to antigen, AID expression is limited to a short window during which the $\mathrm{B}$ cell is diversifying its $\operatorname{IgV}$ regions in the germinal center reaction. AID expression is normally extinguished during plasmacytic differentiations, thereby limiting the exposure of $\mathrm{B}$ cells to this potentially mutagenic enzyme. In contrast, constitutive AID expression in ABC DLBCL may expose the genome of this tumor type to repeated mutation and DNA strand breaks. Sequence analysis of the intra-S $\mu$ deletions in ABC DLBCL revealed that AID was constitutively active in the malignant clone over many generations. These $S \mu$ regions contained as many as 19 independent deletions that were interspersed with somatic mutations targeted at AID hot spots. This remarkably high frequency of switch region deletions is unprecedented in analyses of switch regions from normal mouse B cells. These deletions and mutations could be organized into lineage diagrams that imply that AID-dependent single-stranded and double-stranded breaks were being generated over many generations of the ABC DLBCL clones. GCB DLBCLs had aberrant CSR events less commonly, but in two patient samples we identified ongoing deletions, mutations, and duplications in $\mathrm{S} \mu$. GCB DLBCLs expressed AID mRNA at levels that were lower than in ABC DLBCL but still higher than in mantle cell lymphoma, small lymphocytic lymphoma, and MALT lymphoma (Fig. 3 A). Because AID is likely to participate in the $S \mu$ abnormalities, the level of AID in GCB DLBCL is apparently sufficient for aberrant CSR. Nonetheless, the significantly higher AID expression in ABC DLBCL may contribute to the higher frequency of $S \mu$ deletions in this DLBCL subtype.

However, none of the PMBL samples showed intra-S $\mu$ deletions despite having AID expression levels that were comparable to those in ABC DLBCLs. Therefore, it is unlikely that high AID expression is the only molecular abnormality that contributes to aberrant CSR in ABC DLBCL. The fact that intra-switch deletions predominated over legitimate switch recombination suggests that ABC DLBCLs may have defects in the process of "synapsis" that brings two different switch regions together before recombination (17). Genetic deficiency in three DNA repair proteins, H2AX, 53BP1, and ataxia-teleangiectasia, reduces legitimate CSR in mouse B cells while allowing intra-S $\mu$ deletions to occur $(25,27,37,41,42)$, leading to the hypothesis that these proteins may facilitate the formation of a protein lattice that physically links switch regions that have sustained AIDinduced double-stranded breaks. Thus, it is possible that ABC 
DLBCLs in contrast to PMBL may have a genetic or functional deficiency in one of these DNA repair proteins.

One of the most unanticipated findings in the present study was the insertion of foreign DNA segments within the $\mathrm{S} \mu$ region of one ABC DLBCL case, which may reflect a dysregulation of CSR that predisposes this lymphoma type to chromosomal translocations. The inserted DNA segments originated from three chromosomes and were ligated in tandem without homologous sequences at the junctions, suggesting that they were joined by the NHEJ mechanism. These insertions have not been reported in switch regions from normal B cells (37), further supporting the hypothesis that CSR is dysregulated in ABC DLBCL. However, it has been published that in one case of MALT lymphoma, a 336-bp segment from the ARNT2 gene on chromosome 15 was inserted into $\mathrm{S} \mu$, suggesting that this aberrant CSR mechanism also occurs in other lymphoma types (34). The molecular mechanism responsible for the blunt end insertion of foreign DNA into switch region remains unknown.

We speculate that the extraordinary frequency of intraswitch deletions and the insertions of ectopic DNA into $S \mu$ in ABC DLBCLs may predispose these lymphomas to chromosomal translocations. In the S $\gamma 3-S P I B$ translocation in the OCI-Ly3 cell line, the translocated switch region had also sustained an intra-switch deletion, raising the possibility that these events might be mechanistically linked. By the Southern blot assay, 56\% of ABC DLBCLs had illegitimate CSR events, and these events were less frequent in the other DLBCL subtypes and in MALT lymphoma. At least some of these illegitimate CSR events represent chromosomal translocations as we were able to identify illegitimate switch translocations involving the BCL6, MYC, and SPIB genes in ABC DLBCLs. Also, all cell lines that had illegitimate CSR events in the Southern blot assay had IgH translocations that were demonstrable by FISH. AID is a likely candidate to mediate these chromosomal translocations because it has been shown to be required for spontaneous $M Y C / I g H$ translocations that occur in IL-6-transgenic (29), 53BP1-/- (30), and $H 2 A X^{-1-}$ (43) mice. Compared with resting blood mature $\mathrm{B}$ cells, ABC DLBCLs have 26-fold higher AID mRNA expression (unpublished data). In this regard, it is notable that ectopic overexpression of $A I D$ in mouse $\mathrm{B}$ cells by $\sim 10$-fold was sufficient to cause chromosomal translocations linking the MYC locus to the $I g H$ locus (30). Thus, it is certainly plausible that the high expression of AID in ABC DLBCL contributes to the higher frequency of intra-S $\mu$ deletion and illegitimate CSR in this lymphoma type. AID could contribute to an initiating chromosomal translocation event involving a potent oncogene such as $M Y C$ and BCL6 or could act later in malignant transformation to generate translocations that drive clonal progression. However, because PMBL and ABC DLBCL express AID mRNA at approximately equivalent levels, it is likely that ABC DLBCLs have additional mechanisms that deregulate CSR given the fact that this lymphoma type has higher intra-S $\mu$ and intra-S $\gamma$ deletions and higher illegitimate CSR events than PMBL.
The novel switch translocation involving the SPIB gene that we identified in the ABC cell line OCI-Ly3 highlights the potential biological importance of the switch translocations in ABC DLBCL. SPIB is an Ets family transcription factor that is expressed exclusively in mature B cells, T cell progenitors, and plasmacytoid dendritic cells $(44,45)$. SPIB is required for full $\mathrm{B}$ cell receptor signaling and $\mathrm{T}$ cell-dependent antibody responses (46). SPIB is central to the germinal center reaction because germinal centers in SPIB-deficient mice are smaller in size, persist for a shorter duration after immunization, and contain more apoptotic cells than those in wild-type animals (46). The importance of SPIB as a regulator of mature B cell function is also emphasized by the fact that it is directly repressed by Blimp-1 during plasmacytic differentiation (14). Interestingly, SPIB is significantly more highly expressed in ABC DLBCLs than in GCB DLBCLs (4). This fact together with the translocation of SPIB in the ABC DLBCL cell line OCI-Ly3 suggests that SPIB may play an important role in the pathophysiology of this lymphoma subtype. More generally, we believe that the present study indicates that a comprehensive identification of switch translocations in ABC DLBCL is likely to uncover additional oncogenic pathways in this lymphoma type.

\section{MATERIALS AND METHODS}

Patient samples. Tumor biopsy specimens, which have previously been analyzed by gene expression profiling $(4,5)$, were obtained before treatment from 92 patients with DLBCL and 17 patients with MALT lymphoma. All samples were studied according to a protocol approved by the NCI Institutional Review Board.

Culture of lymphoma cell lines. Lymphoma cell lines were cultured in RPMI, 10\% FBS, except for OCI-Ly1, OCI-Ly2, OCI-Ly3, OCI-Ly4, OCI-Ly7, OCI-Ly8, and OCI-Ly10, which were cultured in Iscove's modified Dulbecco medium with 20\% human plasma.

Southern blot analysis of switch regions. Genomic DNA from cell lines and patient samples was extracted with the DNeasy Tissue kit (QIAGEN) according to the manufacturer's instructions. $10 \mu \mathrm{g}$ of genomic DNA was digested with appropriate restriction enzymes, fractionated on a $0.8 \%$ agarose gel, denatured with a buffer consisting of $0.5 \mathrm{~N} \mathrm{NaOH}, 0.6 \mathrm{M}$ $\mathrm{NaCl}$, neutralized with $1 \mathrm{M}$ Tris-HCL/0.6 M NaCL, and transferred with $20 \times$ SSC to nylon membranes (PerkinElmer). Two radiolabeled probes were created by PCR for each Ig switch region, binding either $5^{\prime}$ or $3^{\prime}$ of the switch region (Fig. 1) as described previously $(28,33)$. Filters were hybridized overnight with the radiolabeled probes at $42^{\circ} \mathrm{C}$ in $1 \mathrm{M} \mathrm{NaCl}$, $50 \mathrm{mM}$ Tris-HCL, pH 7.4, 40\% formamide, 10\% dextran sulfate, 1\% SDS, and $100 \mu \mathrm{g} / \mathrm{ml}$ salmon sperm DNA. The filters were subsequently washed in $2 \times \mathrm{SSC} / 0.1 \% \mathrm{SDS}$ at room temperature and then in $0.1 \times \mathrm{SSC} / 0.1 \%$ SDS at $52-60^{\circ} \mathrm{C}$, followed by exposure to XAR films (Kodak) at $-70^{\circ} \mathrm{C}$ with an intensifying screen.

The Chi-squared test was used for statistical analysis of the difference in CSR events between lymphoma types.

Amplification and sequencing of aberrant $\mathrm{S} \mu$ regions $M Y C, B C L 6$, and SPIB. The $\mathrm{S} \mu$ region was amplified by PCR using primers binding $5^{\prime}$ and $3^{\prime}$ outside of $\mathrm{S} \mu$ (M-F1, GGAGGGGATGCTCCGGGAAGGTGG; M-R1, CGAGGCAGCCAACGGCCACGC). The PCR was performed with TaKaRa La Taq polymerase (Takara Bio, Inc.) using the following conditions: $94^{\circ} \mathrm{C}$ for $1 \mathrm{~min}$, followed by 33 cycles of denaturation, $30 \mathrm{~s}$ at $94^{\circ} \mathrm{C}$; annealing, $30 \mathrm{~s}$ at $68^{\circ} \mathrm{C}$; extension, $6 \mathrm{~min}$ at $72^{\circ} \mathrm{C}$; and a final extension for 
$10 \mathrm{~min}$ at $72^{\circ} \mathrm{C}$. The PCR products were visualized by electrophoresis on an $0.8 \%$ agarose gel and ethidium bromide staining. PCR products were cloned into the Topo XL cloning vector (Invitrogen) and sequenced (BigDye sequencing system; Applied Biosystems).

The PCR reactions for detection of translocations involving the $\mathrm{S} \mu$ and Sy region and MYC or BCL6 were performed as described previously (47). The PCR reactions to confirm a translocation involving the $S \gamma$ region and SPIB were performed applying one of the SPIB primers (GGGGCCCTCACTTACTGTG or AACCAGAAGGGGCAAGAGAC) and one of the Sy primers (GACCAGTGGACACTGTTCTCAGATGG or CCTCCAAGGCCCTTTTCTTCTGTG; reference 48) using the following conditions: $95^{\circ} \mathrm{C}$ for $10 \mathrm{~min}$, followed by 40 cycles of denaturation, $30 \mathrm{~s}$ at $95^{\circ} \mathrm{C}$; annealing, $30 \mathrm{~s}$ at $65^{\circ} \mathrm{C}$; extension, $3 \mathrm{~min}$ at $72^{\circ} \mathrm{C}$, and final extension for $10 \mathrm{~min}$ at $72^{\circ} \mathrm{C}$. The PCR products were visualized by electrophoresis on an $0.8 \%$ agarose gel with ethidium bromide staining, and the PCR product was subsequently sequenced.

FISH. FISH for detection of $\operatorname{IgH}$ translocations with $\mathrm{C}_{\mathrm{H}} \mathrm{BAC}$ and $\mathrm{V}_{\mathrm{H}}$ cosmid probes was performed as described previously (49). For the analysis of SPIB translocation, BAC clones CTB-191K22, CTD-2545M3, and CTD$2560 \mathrm{~K} 21$ as well as a whole chromosome painting probe for chromosome 19 were used. Isolation and labeling of BAC DNA and FISH were performed as described previously (50). MYC and BCL6 translocations were detected using break-apart probes (Vysis probes; Abbott).

Southern blot analysis of SPIB. $10 \mu \mathrm{g}$ of genomic DNA was digested with SphI, and Southern blotting was performed as described above. A radiolabeled 332-bp probe binding $\sim 200$ bp $3^{\prime}$ of the SPIB start codon was created by PCR, and hybridization, washing, and exposure was performed as described above.

Gene expression profiling. RNA was extracted from the biopsy specimens as described previously (3) and was profiled for gene expression with the use of "Lymphochip" DNA microarrays (51) or Affymetrix U133A and U133B oligonucleotide microarrays. The primary gene expression profiling data are available from the Gene Expression Omnibus of the National Center for Biotechnology Information (www.ncbi.nlm.nih.gov/geo) through GEO accession number GSE4732.

Online supplemental material. Table S1 and S2 contain the complete results of the analysis of class switch recombination by Southern blot in DLBCL cell lines and DLBCL and MALT patient samples. Fig. S1 shows lineage models of $\mathrm{S} \mu$ alterations in three ABC DLBCL patient samples (cases 756, 692, and 645) and two GCB DLBCL patient samples (cases 742 and 459). Fig. S2 displays sequence analysis of the $5^{\prime}$ end of $S \mu$ deletion in ABC DLBCL case 756 . The online supplemental material is available at http://www.jem.org/cgi/content/full/jem.20062041/DC1.

We wish to thank Claudia Becher for expert technical assistance.

This research was supported by the Intramural Research Program of the $\mathrm{NIH}, \mathrm{NCl}$, Center for Cancer Research, and an NCl Director's Challenge grant (U01-CA84967). G. Lenz was also supported by a research grant of the German Research Foundation (DFG), and R. Siebert was supported by the Deutsche Krebshilfe and the Kinder-KrebsInitiative Buchholz Holm-Seppensen. This project was performed under the auspices of the Lymphoma/Leukemia Molecular Profiling Project of the NCl.

The authors have no conflicting financial interests.

Submitted: 22 September 2006

Accepted: 31 January 2007

\section{REFERENCES}

1. 1997. A clinical evaluation of the International Lymphoma Study Group classification of non-Hodgkin's lymphoma. The Non-Hodgkin's Lymphoma Classification Project. Blood. 89:3909-3918.

2. Coiffier, B. 200 1. Diffuse large cell lymphoma. Curr. Opin. Oncol. 13:325-334.
3. Alizadeh, A.A., M.B. Eisen, R.E. Davis, C. Ma, I.S. Lossos, A Rosenwald, J.C. Boldrick, H. Sabet, T. Tran, X. Yu, et al. 2000. Distinct types of diffuse large B-cell lymphoma identified by gene expression profiling. Nature. 403:503-511.

4. Rosenwald, A., G. Wright, W.C. Chan, J.M. Connors, E. Campo, R.I. Fisher, R.D. Gascoyne, H.K. Muller-Hermelink, E.B. Smeland, J.M Giltnane, et al. 2002. The use of molecular profiling to predict survival after chemotherapy for diffuse large-B-cell lymphoma. N. Engl. J. Med. 346:1937-1947.

5. Rosenwald, A., G. Wright, K. Leroy, X. Yu, P. Gaulard, R.D Gascoyne, W.C. Chan, T. Zhao, C. Haioun, T.C. Greiner, et al. 2003. Molecular diagnosis of primary mediastinal B cell lymphoma identifies a clinically favorable subgroup of diffuse large B cell lymphoma related to Hodgkin lymphoma. J. Exp. Med. 198:851-862.

6. Wright, G., B. Tan, A. Rosenwald, E.H. Hurt, A. Wiestner, and L.M. Staudt. 2003. A gene expression-based method to diagnose clinically distinct subgroups of diffuse large B cell lymphoma. Proc. Natl. Acad. Sci. USA. 100:9991-9996.

7. Savage, K.J., S. Monti, J.L. Kutok, G. Cattoretti, D. Neuberg, L. De Leval, P. Kurtin, P. Dal Cin, C. Ladd, F. Feuerhake, et al. 2003. The molecular signature of mediastinal large B-cell lymphoma differs from that of other diffuse large B-cell lymphomas and shares features with classical Hodgkin lymphoma. Blood. 102:3871-3879.

8. Staudt, L.M., and S. Dave. 2005. The biology of human lymphoid malignancies revealed by gene expression profiling. Adv. Immunol. 87:163-208.

9. Phan, R.T., and R. Dalla-Favera. 2004. The BCL6 proto-oncogene suppresses p53 expression in germinal-centre B cells. Nature. 432:635-639.

10. Shaffer, A.L., X. Yu, Y. He, J. Boldrick, E.P. Chan, and L.M. Staudt. 2000. BCL-6 represses genes that function in lymphocyte differentiation, inflammation, and cell cycle control. Immunity. 13:199-212.

11. Shaffer, A.L., M. Shapiro-Shelef, N.N. Iwakoshi, A.-H. Lee, S.-B. Qian, H. Zhao, X. Yu, L. Yang, B.K. Tan, A. Rosenwald, et al. 2004 $\mathrm{XBP} 1$, downstream of Blimp-1, expands the secretory apparatus and other organelles, and increases protein synthesis in plasma cell differentiation. Immunity. 21:81-93.

12. Tam, W., M. Gomez, A. Chadburn, J.W. Lee, W.C. Chan, and D.M Knowles. 2006. Mutational analysis of PRDM1 indicates a tumor-suppressor role in diffuse large B-cell lymphomas. Blood. 107:4090-4100.

13. Pasqualucci, L., M. Compagno, J. Houldsworth, S. Monti, A. Grunn, S.V. Nandula, J.C. Aster, V.V. Murty, M.A. Shipp, and R. Dalla-Favera. 2006. Inactivation of the PRDM1/BLIMP1 gene in diffuse large B cell lymphoma. J. Exp. Med. 203:311-317.

14. Shaffer, A.L., K.I. Lin, T.C. Kuo, X. Yu, E.M. Hurt, A. Rosenwald, J.M. Giltnane, L. Yang, H. Zhao, K. Calame, and L.M. Staudt. 2002. Blimp-1 orchestrates plasma cell differentiation by extinguishing the mature B cell gene expression program. Immunity. 17:51-62.

15. Copie-Bergman, C., A. Plonquet, M.A. Alonso, M.L. Boulland, J. Marquet, M. Divine, P. Moller, K. Leroy, and P. Gaulard. 2002. MAL expression in lymphoid cells: further evidence for MAL as a distinct molecular marker of primary mediastinal large B-cell lymphomas. Mod. Pathol. 15:1172-1180.

16. Manis, J.P., M. Tian, and F.W. Alt. 2002. Mechanism and control of class-switch recombination. Trends Immunol. 23:31-39.

17. Chaudhuri, J., and F.W. Alt. 2004. Class-switch recombination: interplay of transcription, DNA deamination and DNA repair. Nat. Rev. Immunol. 4:541-552.

18. Muramatsu, M., V.S. Sankaranand, S. Anant, M. Sugai, K. Kinoshita, N.O. Davidson, and T. Honjo. 1999. Specific expression of activation-induced cytidine deaminase (AID), a novel member of the RNAediting deaminase family in germinal center B cells. J. Biol. Chem. 274:18470-18476.

19. Muramatsu, M., K. Kinoshita, S. Fagarasan, S. Yamada, Y. Shinkai, and T. Honjo. 2000. Class switch recombination and hypermutation require activation-induced cytidine deaminase (AID), a potential RNA editing enzyme. Cell. 102:553-563.

20. Longerich, S., U. Basu, F. Alt, and U. Storb. 2006. AID in somatic hypermutation and class switch recombination. Curr. Opin. Immunol. 18:164-174. 
21. Arakawa, H., J. Hauschild, and J.M. Buerstedde. 2002. Requirement of the activation-induced deaminase (AID) gene for immunoglobulin gene conversion. Science. 295:1301-1306.

22. Revy, P., T. Muto, Y. Levy, F. Geissmann, A. Plebani, O. Sanal, N. Catalan, M. Forveille, R. Dufourcq-Labelouse, A. Gennery, et al. 2000. Activation-induced cytidine deaminase (AID) deficiency causes the autosomal recessive form of the Hyper-IgM syndrome (HIGM2). Cell. 102:565-575.

23. Honjo, T., H. Nagaoka, R. Shinkura, and M. Muramatsu. 2005. AID to overcome the limitations of genomic information. Nat. Immunol. 6:655-661.

24. Petersen, S., R. Casellas, B. Reina-San-Martin, H.T. Chen, M.J. Difilippantonio, P.C. Wilson, L. Hanitsch, A. Celeste, M. Muramatsu, D.R. Pilch, et al. 2001. AID is required to initiate Nbs1/gammaH2AX focus formation and mutations at sites of class switching. Nature. 414:660-665.

25. Reina-San-Martin, B., H.T. Chen, A. Nussenzweig, and M.C. Nussenzweig. 2004. ATM is required for efficient recombination between immunoglobulin switch regions. J. Exp. Med. 200:1103-1110.

26. Schrader, C.E., E.K. Linehan, S.N. Mochegova, R.T. Woodland, and J. Stavnezer. 2005. Inducible DNA breaks in Ig S regions are dependent on AID and UNG. J. Exp. Med. 202:561-568.

27. Manis, J.P., J.C. Morales, Z. Xia, J.L. Kutok, F.W. Alt, and P.B. Carpenter. 2004. 53BP1 links DNA damage-response pathways to immunoglobulin heavy chain class-switch recombination. Nat. Immunol. 5:481-487.

28. Bergsagel, P.L., M. Chesi, E. Nardini, L.A. Brents, S.L. Kirby, and W.M. Kuehl. 1996. Promiscuous translocations into immunoglobulin heavy chain switch regions in multiple myeloma. Proc. Natl. Acad. Sci. USA. 93:13931-13936.

29. Ramiro, A.R., M. Jankovic, T. Eisenreich, S. Difilippantonio, S. Chen-Kiang, M. Muramatsu, T. Honjo, A. Nussenzweig, and M.C. Nussenzweig. 2004. AID is required for c-myc/IgH chromosome translocations in vivo. Cell. 118:431-438.

30. Ramiro, A.R., M. Jankovic, E. Callen, S. Difilippantonio, H.T. Chen, K.M. McBride, T.R. Eisenreich, J. Chen, R.A. Dickins, S.W. Lowe, et al. 2006. Role of genomic instability and p53 in AID-induced c-mycIgh translocations. Nature. 440:105-109.

31. Sciammas, R., A.L. Shaffer, J.H. Schatz, H. Zhao, L.M. Staudt, and H Singh. 2006. Graded expression of interferon regulatory factor-4 coordinates isotype switching with plasma cell differentiation. Immunity. 25:225-236.

32. Klein, U., S. Casola, G. Cattoretti, Q. Shen, M. Lia, T. Mo, T. Ludwig, K. Rajewsky, and R. Dalla-Favera. 2006. Transcription factor IRF4 controls plasma cell differentiation and class-switch recombination. Nat. Immunol. 7:773-782

33. Chesi, M., P.L. Bergsagel, L.A. Brents, C.M. Smith, D.S. Gerhard, and W.M. Kuehl. 1996. Dysregulation of cyclin D1 by translocation into an IgH gamma switch region in two multiple myeloma cell lines. Blood. 88:674-681.

34. Nardini, E., A. Aiello, R. Giardini, M.I. Colnaghi, S. Menard, and A. Balsari. 2000. Detection of aberrant isotype switch recombination in low-grade and high-grade gastric MALT lymphomas. Blood. 95:1032-1038

35. Dave, S.S., K. Fu, G.W. Wright, L.T. Lam, P. Kluin, E.J. Boerma, T.C. Greiner, D.D. Weisenburger, A. Rosenwald, G. Ott, et al 2006. Molecular diagnosis of Burkitt's lymphoma. N. Engl. J. Med. 354:2431-2442

36. Dudley, D.D., J.P. Manis, A.A. Zarrin, L. Kaylor, M. Tian, and F.W. Alt. 2002. Internal IgH class switch region deletions are position-independent and enhanced by AID expression. Proc. Natl. Acad. Sci. USA. 99:9984-9989.
37. Reina-San-Martin, B., S. Difilippantonio, L. Hanitsch, R.F. Masilamani, A. Nussenzweig, and M.C. Nussenzweig. 2003. H2AX is required for recombination between immunoglobulin switch regions but not for intra-switch region recombination or somatic hypermutation. J. Exp. Med. 197:1767-1778

38. Davis, R.E., K.D. Brown, U. Siebenlist, and L.M. Staudt. 2001. Constitutive nuclear factor $\mathrm{\kappa B}$ activity is required for survival of activated $\mathrm{B}$ cell-like diffuse large B cell lymphoma cells. J. Exp. Med. 194:1861-1874.

39. Lam, L.T., R.E. Davis, J. Pierce, M. Hepperle, Y. Xu, M. Hottelet, Y. Nong, D. Wen, J. Adams, L. Dang, and L.M. Staudt. 2005. Small molecule inhibitors of IkB-kinase are selectively toxic for subgroups of diffuse large B cell lymphoma defined by gene expression profiling. Clin. Cancer Res. 11:28-40.

40. Pasqualucci, L., R. Guglielmino, J. Houldsworth, J. Mohr, S Aoufouchi, R. Polakiewicz, R.S. Chaganti, and R. Dalla-Favera. 2004 Expression of the AID protein in normal and neoplastic B cells. Blood. 104:3318-3325.

41. Lumsden, J.M., T. McCarty, L.K. Petiniot, R. Shen, C. Barlow, T.A. Wynn, H.C. Morse III, P.J. Gearhart, A. Wynshaw-Boris, E.E. Max, and R.J. Hodes. 2004. Immunoglobulin class switch recombination is impaired in Atm-deficient mice. J. Exp. Med. 200:1111-1121.

42. Reina-San-Martin, B., J. Chen, A. Nussenzweig, and M.C. Nussenzweig. 2007. Enhanced intra-switch region recombination during immunoglobulin class switch recombination in 53BP1(-/-) B cells. Eur. J. Immunol. 37:235-239.

43. Franco, S., M. Gostissa, S. Zha, D.B. Lombard, M.M. Murphy, A.A. Zarrin, C. Yan, S. Tepsuporn, J.C. Morales, M.M. Adams, et al. 2006 H2AX prevents DNA breaks from progressing to chromosome breaks and translocations. Mol. Cell. 21:201-214.

44. Schotte, R., M.C. Rissoan, N. Bendriss-Vermare, J.M. Bridon, T. Duhen, K. Weijer, F. Briere, and H. Spits. 2003. The transcription factor Spi-B is expressed in plasmacytoid DC precursors and inhibits T-, B-, and NK-cell development. Blood. 101:1015-1023.

45. Su, G.H., H.S. Ip, B.S. Cobb, M.M. Lu, H.M. Chen, and M.C. Simon 1996. The Ets protein Spi-B is expressed exclusively in B cells and T cells during development. J. Exp. Med. 184:203-214.

46. Su, G.H., H.M. Chen, N. Muthusamy, L.A. Garrett-Sinha, D. Baunoch, D.G. Tenen, and M.C. Simon. 1997. Defective B cell receptor-mediated responses in mice lacking the Ets protein, Spi-B. EMBOJ. 16:7118-7129.

47. Akasaka, T., M. Muramatsu, H. Ohno, I. Miura, E. Tatsumi, S Fukuhara, T. Mori, and M. Okuma. 1996. Application of long-distance polymerase chain reaction to detection of junctional sequences created by chromosomal translocation in mature B-cell neoplasms. Blood. 88:985-994

48. Sonoki, T., T.G. Willis, D.G. Oscier, E.L. Karran, R. Siebert, and M.J. Dyer. 2004. Rapid amplification of immunoglobulin heavy chain switch (IGHS) translocation breakpoints using long-distance inverse PCR. Leukemia. 18:2026-2031.

49. Shou, Y., M.L. Martelli, A. Gabrea, Y. Qi, L.A. Brents, A. Roschke, G. Dewald, I.R. Kirsch, P.L. Bergsagel, and W.M. Kuehl. 2000. Diverse karyotypic abnormalities of the c-myc locus associated with c-myc dysregulation and tumor progression in multiple myeloma. Proc. Natl. Acad. Sci. USA. 97:228-233.

50. Martin-Subero, J.I., I. Chudoba, L. Harder, S. Gesk, W. Grote, F.J. Novo, M.J. Calasanz, and R. Siebert. 2002. Multicolor-FICTION: expanding the possibilities of combined morphologic, immunophenotypic, and genetic single cell analyses. Am. J. Pathol. 161:413-420.

51. Alizadeh, A., M. Eisen, R.E. Davis, C. Ma, H. Sabet, T. Tran, J. Powell, L. Yang, G. Marti, T. Moore, et al. 1999. The lymphochip: a specialized cDNA microarray for the genomic-scale analysis of gene expression in normal and malignant lymphocytes. Cold Spring Harb. Symp. Quant. Biol. $64: 71-78$ 